\title{
Quantum Information Processing in Optical Lattices and Magnetic Microtraps
}

\author{
Philipp Treutlein*, Tilo Steinmetz $₫$ Yves Colombe \\ Markus Greiner Olaf Mandel 5 Arthur Widera** Tim Rom** Immanuel Bloch*** and Theodor W. Hänsch \\ Max-Planck-Institut für Quantenoptik und Sektion Physik der \\ Ludwig-Maximilians-Universität, Schellingstr. 4, 80799 München, Germany
}

(Dated: 18 May 2006)

\begin{abstract}
We review our experiments on quantum information processing with neutral atoms in optical lattices and magnetic microtraps.

Atoms in an optical lattice in the Mott insulator regime serve as a large qubit register. A spindependent lattice is used to split and delocalize the atomic wave functions in a controlled and coherent way over a defined number of lattice sites. This is used to experimentally demonstrate a massively parallel quantum gate array, which allows the creation of a highly entangled many-body cluster state through coherent collisions between atoms on neighbouring lattice sites.

In magnetic microtraps on an atom chip, we demonstrate coherent manipulation of atomic qubit states and measure coherence lifetimes exceeding one second at micron-distance from the chip surface. We show that microwave near-fields on the chip can be used to create state-dependent potentials for the implementation of a quantum controlled phase gate with these robust qubit states. For single atom detection and preparation, we have developed high finesse fiber Fabry-Perot cavities and integrated them on the atom chip. We present an experiment in which we detected a very small number of cold atoms magnetically trapped in the cavity using the atom chip.
\end{abstract}

\section{Contents}

$\begin{array}{ll}\text { Introduction } & 1\end{array}$

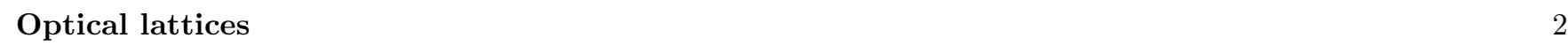

Preparation of a qubit register $\quad 2$

A quantum convever belt for neutral atoms

Controlled collisions

Magnetic microtraps

5

Qubit states on the atom chip $\quad 6$

$\begin{array}{lr}\text { State-dependent microwave potentials } & 8\end{array}$

\begin{tabular}{lr}
\hline Qubit readout in microtraps & 10
\end{tabular}

$\begin{array}{ll}\text { Stable fiber Fabrv-Perot cavities } & 11\end{array}$

FFP cavitv fabrication and performance $\quad 12$

On-chip atom detection with a FFP cavity 12

$\begin{array}{lr}\text { Single atom preparation } & 14\end{array}$

$\begin{array}{lr}\text { Conclusion } & 14\end{array}$

$\begin{array}{ll}\text { References } & 14\end{array}$

\section{INTRODUCTION}

Neutral atoms present two essential advantages for quantum information processing (QIP). They are relatively weakly coupled to the environment, so that decoherence can be controlled better than in most other systems. Furthermore, complete control of all quantum-mechanical degrees of freedom is already a reality, and is used in experiments with great success, most notably in Bose-Einstein condensation.

\footnotetext{
* Corresponding author. E-mail: philipp.treutlein@physik.lmu.de, Phone: +4989 2180-3937, Fax: +4989 2180-3938
} 
Theoretical approaches have been developed to use atoms in well-defined states of controllable potentials for creating many-particle entanglement, and qubit operations in particular. Experimentally realizing these proposals is a major challenge and requires new ideas to overcome the subtle problems occuring in real atomic systems. Requirements on stability and control of environmental conditions, such as electric and magnetic stray fields, are equally demanding.

In the theoretical investigations already, two experimental systems emerged as particularly promising embodiments for neutral-atom QIP. Optical lattices allow for a large number of qubits due to their three-dimensional, periodic structure. In magnetic microtraps (atom chips), complex potentials can be realized, and lithographic fabrication techniques enable scalability and modularity in analogy with microelectronics. In the following, we review experimental progress achieved in our group with both systems.

\section{OPTICAL LATTICES}

\section{Preparation of a qubit register}

Starting point for the preparation of the neutral atom qubit register is an atomic Bose-Einstein condensate. This is placed in an artificial crystal of light - a so called optical lattice - which is formed by standing wave laser fields along all three space dimensions. By continuously increasing the lattice depth of the optical potentials, one can drive the system through a quantum phase transition from a superfluid to a Mott insulator 1, 2], where a defined number of atoms is placed on each lattice site (see Fig 1). By controlling the initial total number of atoms and the confinement parameters of the lattice trap, it is possible to have a large connected region to be populated by single atoms on each lattice site. On each of these sites, the atoms occupy the ground state of the trapping potential and their internal state is initialized to a defined state as well.
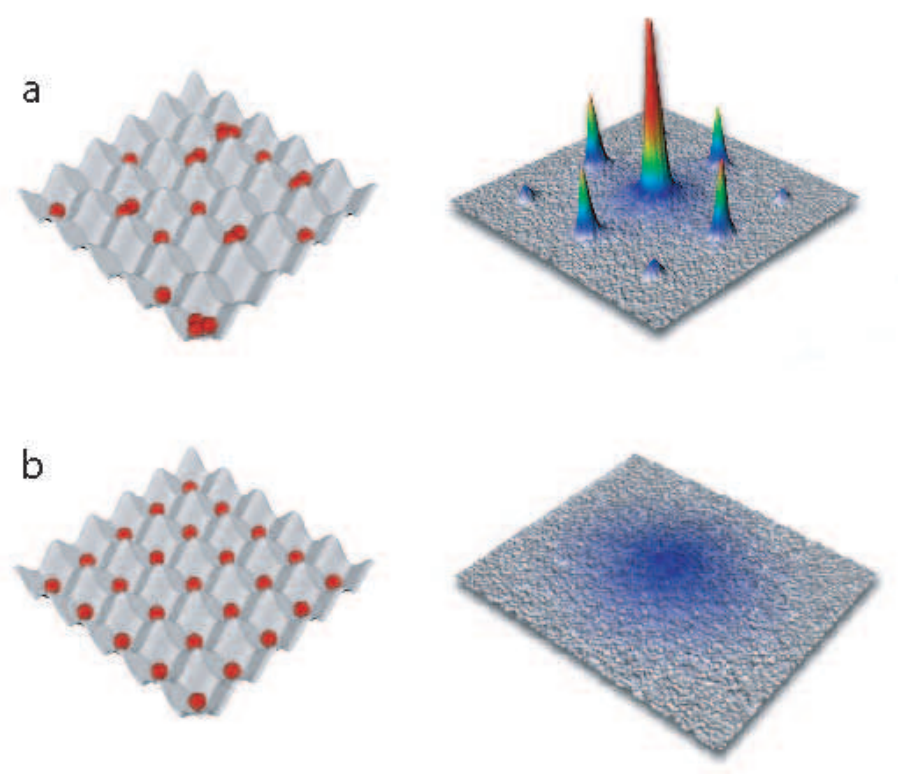

FIG. 1: (a) In the superfluid state of a Bose-Einstein condensate, the underlying atoms can be described as a giant macroscopic matter wave. When such a condensate is released from the periodic potential a multiple matter wave interference pattern is formed due to the phase coherence between the atomic wavefunctions on different lattice sites. In this case the phase of the macroscopic matter wave is well defined. However, the number of atoms at each lattice site fluctuates. (b) In the limit of a Mott insulating state, each lattice site is filled with a fixed number of atoms but the phase of the matter wave field remains uncertain. As a result, no matter wave interference pattern can be seen when the quantum gases are released from the lattice potential. 
(a)

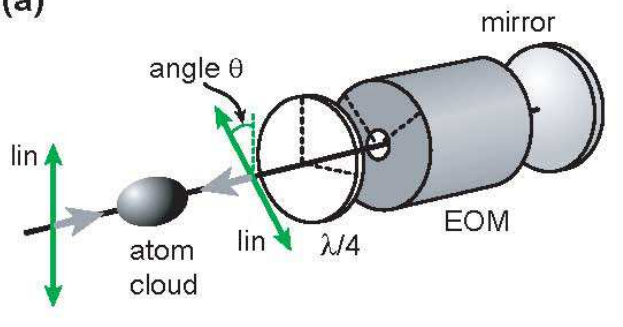

(b)

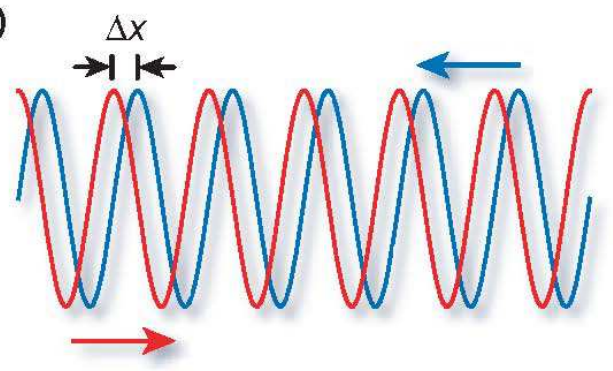

FIG. 2: (a) Schematic experimental setup. A one dimensional optical standing wave laser field is formed by two counterpropagating laser beams with linear polarizations. The polarization angle of the returning laser beam can be adjusted through an electro-optical modulator. The dashed lines indicate the principal axes of the wave plate and the EOM. (b) By increasing the polarization angle $\theta$, one can shift the two resulting $\sigma^{+}$(blue) and $\sigma^{-}$(red) polarized standing waves relative to each other.

\section{A quantum conveyer belt for neutral atoms}

So far the optical potentials used for optical lattices with Bose-Einstein condensates have been mostly independent of the internal ground state of the atom. However, it has been suggested that by using spin-dependent periodic potentials one could bring atoms on different lattice sites into contact and thereby realize fundamental quantum gates $[\underline{3}, 4,[5,6]$, create large scale entanglement [7, 8], excite spin waves [9], study quantum random walks [10] or form a universal quantum simulator to simulate fundamental complex condensed matter physics hamiltonians [11]. Here we show how the wave packet of an atom that is initially localized to a single lattice site can be split and delocalized in a controlled and coherent way over a defined number of lattice sites.

In order to realize a spin dependent transport for neutral atoms in optical lattices, a standing wave configuration formed by two counterpropagating laser beams with linear polarization vectors enclosing an angle $\theta$ has been proposed [3, 7]. Such a standing wave light field can be decomposed into a superposition of a $\sigma^{+}$and $\sigma^{-}$polarized standing wave laser field, giving rise to lattice potentials $V_{+}(x, \theta)=V_{0} \cos ^{2}(k x+\theta / 2)$ and $V_{-}(x, \theta)=V_{0} \cos ^{2}(k x-\theta / 2)$. By changing the polarization angle $\theta$, one can control the separation between the two potentials $\Delta x=\theta / 180^{\circ} \cdot \lambda_{x} / 2$ (see Fig. 2b). When increasing $\theta$, both potentials shift in opposite directions and overlap again when $\theta=n \cdot 180^{\circ}$, with $n$ being an integer. For a spin-dependent transfer, two internal spin states of the atom should be used, where one spin state dominantly experiences the $V_{+}(x, \theta)$ dipole potential and the other spin state mainly experiences the $V_{-}(x, \theta)$ potential. Such a situation can be realized in rubidium by tuning the wavelength of the optical lattice laser to a value of $\lambda_{x}=785 \mathrm{~nm}$ between the fine structure splitting of the rubidium D1 and D2 transition. If an atom is now first placed in a coherent superposition of both internal states $1 / \sqrt{2}(|0\rangle+i|1\rangle)$ and the polarization angle $\theta$ is continuously increased, the spatial wave packet of the atom is split with both components moving in opposite directions.

With such a quantum conveyer belt, atoms have been moved over a defined number of lattice sites. In the experiment a coherent transport of the atoms over a distance of up to 7 lattice sites has been demostrated [12] (see Fig. [3).

\section{Controlled collisions}

In order to realize a controlled interaction between the particles on different lattice sites in a 3D Mott insulating quantum register, the above spin dependent transport sequence can be used. This leads to collisions between neighbouring atoms and can be described through an ensemble of quantum gates acting in parallel [4, 7]. Alternatively, these quantum gates can be described as a controllable quantum Ising interaction [8]:

$$
H_{\mathrm{int}} \propto g(t) \sum_{j} \frac{1+\sigma_{z}^{(j)}}{2} \frac{1-\sigma_{z}^{(j+1)}}{2}
$$

Here $g(t)$ denotes the time dependent coupling constant and $\sigma_{z}^{(j)}$ is the Pauli spin operator acting on an atom at the $j^{t h}$ lattice site. For an interaction phase of $\varphi=2 \pi \times \int_{0}^{t_{\text {hold }}} g(t) d t / h=(2 n+1) \pi$ one obtains a maximally entangled cluster state, whereas for $\varphi=2 n \pi$ one obtains a disentangled state [8]. Here $t_{\text {hold }}$ denotes the time for which the 
(i)

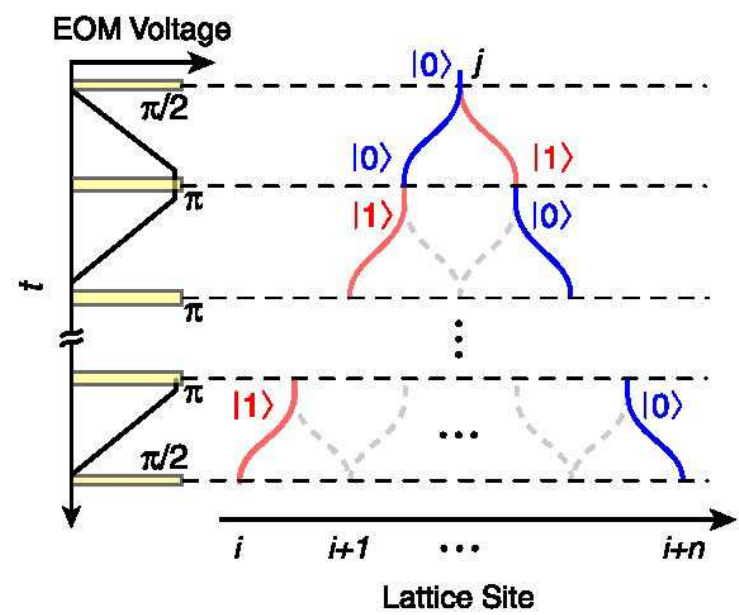

(ii)

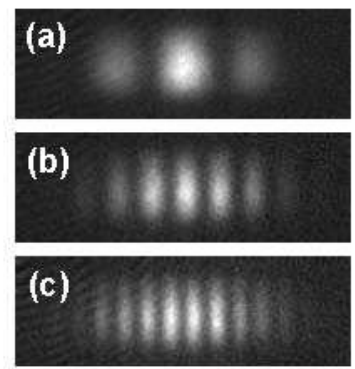

FIG. 3: (i) Schematic sequence used for the quantum conveyer belt. A single atom on lattice site j can be transported over an arbitrary number of lattice sites depending on its spin state (marked as blue and red curves). (ii) This has allowed us to split the wave function of the atom in a coherent way, such that a single atom simultaneously moves to the left and to the right. The coherence of the split wave-packets has been demonstrated in an interference experiment. For larger distances between the split wave-functions, the period of the interference pattern decreases.

atoms are held together at a common site, $h$ is Planck's constant and $n$ is an integer. Let us point out that the creation of such highly entangled states can be achieved in a single lattice shift operational sequence described above and depicted in Fig. 4 independent of the number of atoms to be entangled [7, 8].

A $\pi / 2$ pulse allows us to place the atom in a coherent superposition of the two states $|0\rangle \equiv\left|F=1, m_{F}=-1\right\rangle$ and $|1\rangle \equiv\left|F=2, m_{F}=-2\right\rangle$ within a time of $6 \mu$ s. After creating such a coherent superposition, we use a spin-dependent transfer to split and move the spatial wave function of the atom over half a lattice spacing in two opposite directions depending on its internal state (see Fig. 4). Atoms on neighbouring sites interact for a variable amount of time $t_{\text {hold }}$ that leads to a controlled conditional phase shift of the corresponding many body state. After half of the hold time, a microwave $\pi$ pulse is furthermore applied. This spin-echo type pulse is mainly used to cancel unwanted single particle phase shifts e.g. due to inhomogeneities in the trapping potentials. It does not, however, affect the non-trivial and crucial collisional phase shift due to the interactions between the atoms. After such a controlled collision, the atoms are moved back to their original site. Then a final $\pi / 2$ microwave pulse with variable phase is applied and the atom number in state $|1\rangle$ relative to the total atom number is recorded 13$]$.

For short hold times, where no significant collisional phase shift is acquired, a Ramsey fringe with a high visibility of approx. $50 \%$ is recorded (see Fig. 5). For longer hold times, we notice a strong reduction in the visibility of the Ramsey fringe, with an almost vanishing visibility of approx. $5 \%$ for a hold time of $210 \mu$ s. This hold time corresponds to an acquired collisional phase shift of $\varphi=\pi$ for which we expect a minimum visibility if the system is becoming entangled. For a two-particle system this can be understood by observing the resulting Bell state:

$$
1 / \sqrt{2}\left(|0\rangle_{j}|+\rangle_{j+1}^{\alpha}+|1\rangle_{j}|-\rangle_{j+1}^{\alpha}\right)
$$

after the final $\pi / 2$ pulse of the Ramsey sequence has been applied to the atoms. Here $|+\rangle_{j+1}^{\alpha}$ and $|-\rangle_{j+1}^{\alpha}$ represent two orthogonal superposition states of $|0\rangle$ and $|1\rangle$ for which $\left|\langle 1 \mid+\rangle^{\alpha}\right|^{2}+\left|\langle 1 \mid-\rangle^{\alpha}\right|^{2}=0.5$. A measurement of atoms in state $|1\rangle$ therefore becomes independent of the phase corresponding to a vanishing Ramsey fringe. This indicates that no single particle operation can place all atoms in either spin-state when a maximally entangled state has been created. The disappearance of the Ramsey fringe has been shown to occur not only for a two-particle system, but is a general feature for an arbitrary $N$-particle array of atoms that have been highly entangled with the above experimental sequence [4]. For longer hold times however, the visibility of the Ramsey fringe increases again reaching a maximum of $55 \%$ for a hold time of $450 \mu \mathrm{s}$ (see Fig. 5). Here the system becomes disentangled again, as the collisional phase shift is close to $\varphi=2 \pi$ and the Ramsey fringe is restored with maximum visibility. The timescale of the observed collisional phase evolution is in good agreement with the measurements on the Mott insulator transition of the previous section and ab-initio calculations of the onsite matrix element $U$ [1, 2]. 
(a)

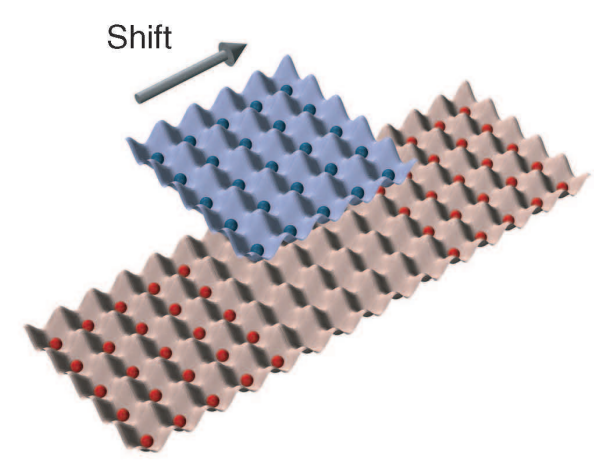

(b)

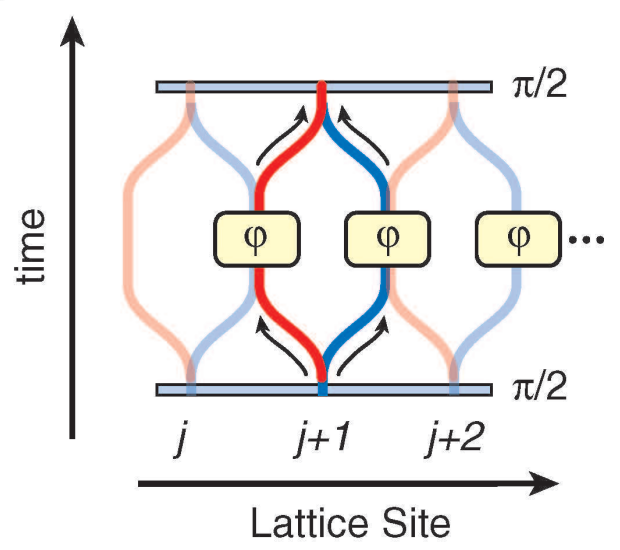

FIG. 4: (a) Controlled interactions between atoms on different lattice sites can be realized with the help of spin-dependent lattice potentials. In such spin dependent potentials, atoms in a, let us say, blue internal state experience a different lattice potential than atoms in a red internal state. These lattices can be moved relative to each other such that two initially separated atoms can be brought into controlled contact with each other. (b) This can be extendended to form a massively parallel quantum gate array. Consider a string of atoms on different lattice sites. First the atoms are placed in a coherent superposition of the two internal states (red and blue). Then spin dependent potentials are used to split each atom such that it simultaneously moves to the right and to the left and is brought into contact with the neighbouring atoms. There both atoms interact and a controlled phase shift $\varphi$ is introduced. After such a controlled collision the atoms are again moved back to their original lattice sites.

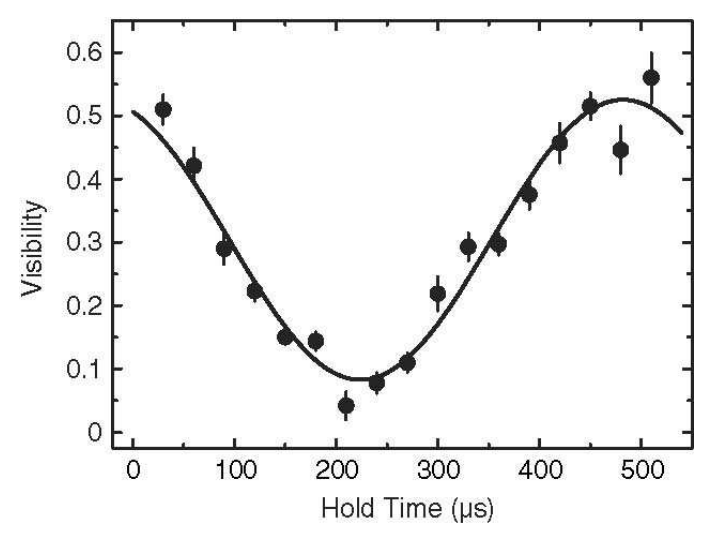

FIG. 5: Visibility of Ramsey fringes vs. hold times on neighbouring lattice sites for the experimental sequence of Fig. 4 The solid line is a sinusoidal fit to the data including an offset and a finite amplitude. Such a sinusoidal behaviour of the visibility vs. the collisional phase shift (determined by the hold time $t_{\text {hold }}$ ) is expected for a Mott insulating state with an occupancy of $\mathrm{n}=1$ atom per lattice site.

\section{MAGNETIC MICROTRAPS}

Atom chips 14, 15] combine many important features of a scalable architecture for quantum information processing [16]: The long coherence lifetimes of qubits based on hyperfine states of neutral atoms [17], accurate control of the coherent evolution of the atoms in tailored micropotentials [18, 19], and scalability of the technology through microfabrication 20, 21] - which allows the integration of many qubits in parallel on the same device while maintaining individual addressability. Furthermore, atom chips offer the exciting perspective of creating interfaces between the atomic qubits and other QIP systems integrated on the same chip, such as photons in optical fiber cavities or solid-state QIP systems located on the chip surface 22]. However, the experimental demonstration of a fundamental two-qubit quantum gate on an atom chip is an important milestone which still has to be reached.

In [23], a first theoretical proposal for a quantum gate on an atom chip was put forward. In this proposal, the gate operation relies on collisional interactions between two atoms in a state-selective potential on the chip. The 
experimental challenge of implementing such a gate can be divided into several steps:

1. A qubit state pair has to be identified which can be manipulated with electromagnetic fields on the atom chip, but still allows for long coherence lifetimes in a realistic experimental situation. In particular, attention has to be paid to decoherence and loss mechanisms induced by the chip surface, which is typically at a distance of only few microns from the atoms.

2. The gate proposed in 23] requires potentials which affect the two qubit states differently in order to achieve conditional logical operations between two atoms. A method to create the required potentials on a chip has to be developed.

3. While Bose-Einstein condensates and thermal ensembles of atoms are routinely manipulated and detected on atom chips, the existing proposals for quantum information processing on atom chips rely on coherent control over single atoms. As a first step towards single atom operation, a single atom detector has to be developed which can be integrated on the atom chip.

4. With a single atom detector available, a method for the deterministic preparation of single neutral atoms in the motional ground state of chip traps with very low occupation probability of excited states has to be found.

In the following, we develop a scenario in which these challenges can be met with atom chips and discuss our experiments towards its realization.

\section{Qubit states on the atom chip}

Two conflicting requirements have to be met by the qubit states $\{|0\rangle,|1\rangle\}$ chosen for QIP on an atom chip. On the one hand, both qubit states have to couple to electromagnetic fields which are used for trapping and manipulating the atoms. In all experiments performed so far, at least a part of the trapping potential is provided by static magnetic fields generated by wires or permanent magnet structures on the atom chip. It is therefore desirable that both $|0\rangle$ and $|1\rangle$ are magnetically trappable states. On the other hand, gate operations with high fidelity are only possible if the coherence lifetimes of the superposition states $\alpha|0\rangle+\beta|1\rangle,\left(|\alpha|^{2}+|\beta|^{2}=1\right)$ are sufficiently long. Long coherence lifetimes are possible if qubit basis states are chosen whose energy difference $h \nu_{10}=E_{|1\rangle}-E_{|0\rangle}$ is robust against noise in realistic experimental situations. In particular, technical fluctuations of magnetic fields are notorious for limiting the coherence lifetime of magnetic-field sensitive qubit states of atoms or ions to a few milliseconds [24]. On atom chips, magnetic near-field noise due to thermally excited currents in the chip wires is an additional fundamental source of decoherence for magnetic field sensitive qubit states 25]. To achieve long coherence lifetimes on atom chips, it is therefore highly desirable to choose a pair of qubit basis states whose energy difference is insensitive to magnetic field fluctuations.

We choose the $\left|F=1, m_{F}=-1\right\rangle \equiv|0\rangle$ and $\left|F=2, m_{F}=+1\right\rangle \equiv|1\rangle$ hyperfine levels of the $5 S_{1 / 2}$ ground state of ${ }^{87} \mathrm{Rb}$ atoms as qubit basis states. The magnetic moments and the corresponding static Zeeman shifts of the two states are approximately equal, leading to a strong common mode suppression of magnetic field induced decoherence. Furthermore, both states experience nearly identical trapping potentials in magnetic traps, thereby avoiding undesired entanglement between internal and external degrees of freedom of the atoms. At a magnetic field of $B_{0} \sim 3.23 \mathrm{G}$, both states experience the same first-order Zeeman shift and the remaining magnetic field dependence of the transition frequency $\nu_{10}$ is minimized [26]. In all of our experiments, we therefore adjust the field in the center of the trap to $B_{0}$.

We have studied the coherence properties of the state pair $\{|0\rangle,|1\rangle\}$ on an atom chip in a series of experiments [17], which we summarize in the following.

The coherence measurements are performed with an ultracold ensemble of atoms, which is prepared in a multi-step sequence involving loading of the microtrap from a mirror-MOT, compression of the trap and evaporative cooling 27]. By the end of this sequence, a thermal atomic ensemble of typically $N_{\text {at }}=1.5 \times 10^{4}$ atoms in state $|0\rangle$ at a temperature of $0.6 \mu \mathrm{K}$ is trapped in a Ioffe-type microtrap. By modulating the currents and offset magnetic fields used to create this trap, the atoms can be placed at distances $d=0-130 \mu \mathrm{m}$ from the chip surface with only small changes in the shape of the magnetic potential. It is advantageous to perform the coherence measurements with a small thermal ensemble instead of a Bose-Einstein condensate, since the higher atomic densities in the condensate would lead to a stronger inhomogeneous collisional broadening of the qubit transition [26].

Single-qubit rotations are implemented by coupling the states $|0\rangle$ and $|1\rangle$ through a two-photon microwave-rf transition as shown in Fig. 6 . The microwave frequency $\nu_{\mathrm{mw}}$ is detuned by $\delta / 2 \pi=1.2 \mathrm{MHz}$ above the $\left|F=2, m_{F}=0\right\rangle$ 
a)

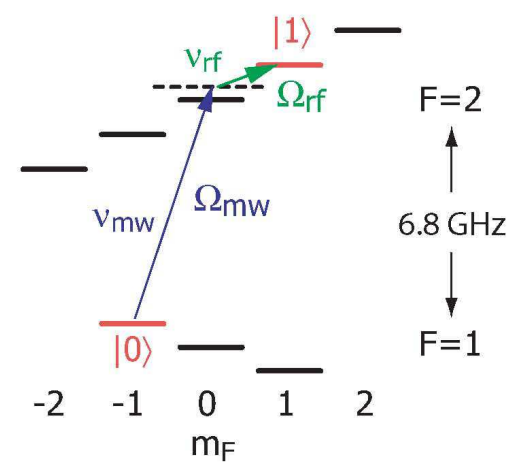

b)

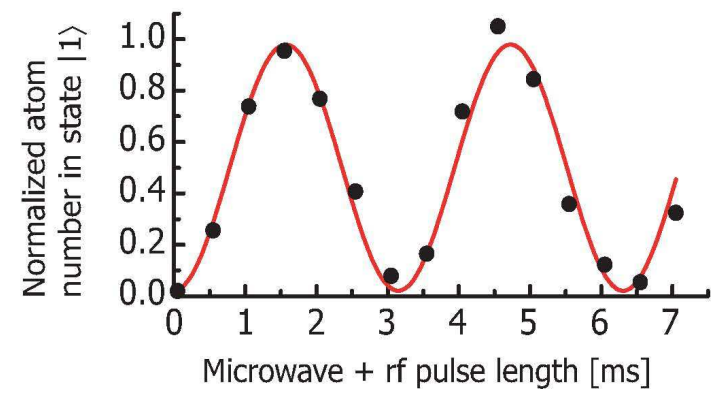

FIG. 6: Single qubit rotations between the states $|0\rangle$ and $|1\rangle$. (a) Ground state hyperfine structure of ${ }^{87} \mathrm{Rb}$ in a weak magnetic field. The first order Zeeman shift of the states $|0\rangle$ and $|1\rangle$ is approximately identical. The two-photon transition $|0\rangle \leftrightarrow|1\rangle$ is driven by a microwave $\nu_{\mathrm{mw}}$ and a radio-frequency $\nu_{\mathrm{rf}}$. $\Omega_{\mathrm{mw}}$ and $\Omega_{\mathrm{rf}}$ are the single-photon Rabi frequencies of the microwave and rf transition, respectively. (b) Two-photon Rabi oscillations recorded as a function of the microwave and rf pulse length. The two-photon Rabi frequency is $\Omega_{2 \mathrm{ph}} / 2 \pi=0.32 \mathrm{kHz}$.

intermediate state and radiated from a sawed-off waveguide outside the vacuum chamber. The radio frequency $\nu_{\mathrm{rf}}$ is either applied to an external coil or to a wire on the chip. $\nu_{\mathrm{mw}}$ and $\nu_{\mathrm{rf}}$ are phase locked to a $10 \mathrm{MHz}$ reference from an ultrastable quartz oscillator. $\Omega_{\mathrm{mw}}$ and $\Omega_{\mathrm{rf}}$ are the single-photon Rabi frequencies of the microwave and $\mathrm{rf}$ transition, respectively. By applying the two-photon drive for a variable time and detecting the number of atoms $N_{1}$ transferred from $|0\rangle$ to $|1\rangle$, we observe Rabi oscillations with a resonant two-photon Rabi frequency of $\Omega_{2 \mathrm{ph}} / 2 \pi=0.32 \mathrm{kHz}$, see Fig. 6b. The maximum transition probability, corresponding to a $\pi$-pulse, is $N_{1} / N_{\text {at }}=95 \pm 5 \%$.

The two-photon Rabi frequency is given by $\Omega_{2 \mathrm{ph}}=\Omega_{\mathrm{mw}} \Omega_{\mathrm{rf}} / 2 \delta$ if $\Omega_{\mathrm{mw}}^{2}, \Omega_{\mathrm{rf}}^{2} \ll \delta^{2}\left[28\right.$, with $\Omega_{\mathrm{mw}} \sim \Omega_{\mathrm{rf}} \sim 2 \pi \times 25 \mathrm{kHz}$ in our experiment. In the present experiment, the two-photon Rabi-frequency is limited by the available microwave power of typically a few watts. Instead of radiating the microwave and rf from antennas outside the vacuum chamber, they can be applied to the atoms much more efficiently by coupling the microwave and rf signal into wires designed as waveguiding structures on the chip. Consider a waveguide on a chip with a characteristic impedance of $Z_{c}=50 \Omega$ carrying a microwave signal of $P=1 \mathrm{~mW}$, corresponding to a microwave current of $I_{\mathrm{mw}}=\sqrt{2 P / Z_{c}}=6.3 \mathrm{~mA}$ on the signal conductor. At a distance of $d=10 \mu \mathrm{m}$ from the signal conductor, the microwave magnetic field amplitude is approximately $B_{\mathrm{mw}} \sim \mu_{0} I_{\mathrm{mw}} /(2 \pi d)=1.3 \mathrm{G}$. The microwave induces a coupling with a single-photon Rabi frequency of the order of $\Omega_{\mathrm{mw}} / 2 \pi \sim \mu_{B} B_{\mathrm{mw}} / h=1.8 \mathrm{MHz}$. This shows that it is advantageous to couple the atomic transitions with microwave and $\mathrm{rf}$ near fields instead of radiation from antennas.

To test for decoherence of the superposition states, we perform Ramsey spectroscopy by applying the following pulse sequence: The atoms in state $|0\rangle$ are held in the trap before a first $\pi / 2$-pulse creates a coherent superposition of $|0\rangle$ and $|1\rangle$. After a time delay $T_{R}$, a second $\pi / 2$-pulse is applied, and the resulting state is probed by detecting the number of atoms transferred to state $|1\rangle$. Ramsey fringes are recorded in the time domain by varying $T_{R}$ while keeping $\delta_{R}=\nu_{\mathrm{mw}}+\nu_{\mathrm{rf}}-\nu_{10}$ fixed $\left(\delta_{R} \ll \nu_{10} \simeq 6.8 \mathrm{GHz}\right)$. Alternatively, Ramsey fringes are recorded in the frequency domain by scanning $\delta_{R}$ while $T_{R}$ remains constant. Loss of coherence of the superposition states can show up in different ways in the Ramsey signal. A spatial variation of $\nu_{10}$ across the atomic ensemble leads to a decay of the contrast of the Ramsey fringes, while temporal fluctuations of $\nu_{10}$ lead to increasing phase noise on the Ramsey oscillation as $T_{R}$ is increased.

Figure 7 shows Ramsey interference in the time domain. The number of atoms detected in state $|1\rangle$ oscillates at the frequency difference $\delta_{R}=6.4 \mathrm{~Hz}$, while the interference contrast decays with a coherence lifetime of $\tau_{c}=2.8 \pm 1.6 \mathrm{~s}$. The measurement shown in Fig. $7 \mathrm{~h}$ was performed at a distance $d=9 \mu \mathrm{m}$ from the room-temperature chip surface. In [26], similar coherence lifetimes are reported for the same state pair, but with atoms in a macroscopic magnetic trap, far away from any material objects. This suggests that atom-surface interactions indeed do not limit the coherence lifetime in our present experiment.

To further probe for surface effects, we study the decoherence of the Ramsey signal as a function of atom-surface distance. Atomic ensembles are prepared in traps at different distances $d$ from the surface. In each trap, we record Ramsey oscillations in the frequency domain for several values of $T_{R}$ and determine the contrast $C\left(T_{R}\right)$ of each oscillation. Figure [7b shows the result of these measurements for $T_{R}=50 \mathrm{~ms}$ and $T_{R}=1 \mathrm{~s}$. Within the experimental error, the contrast does not show a dependence on atom-surface distance for $d=5-130 \mu \mathrm{m}$. Additionally, we have 
a)

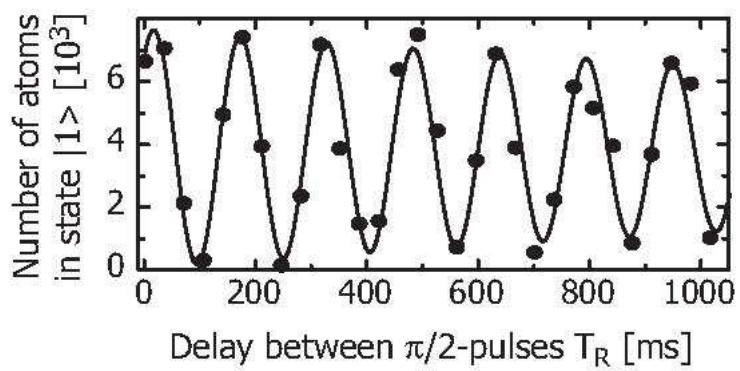

b)

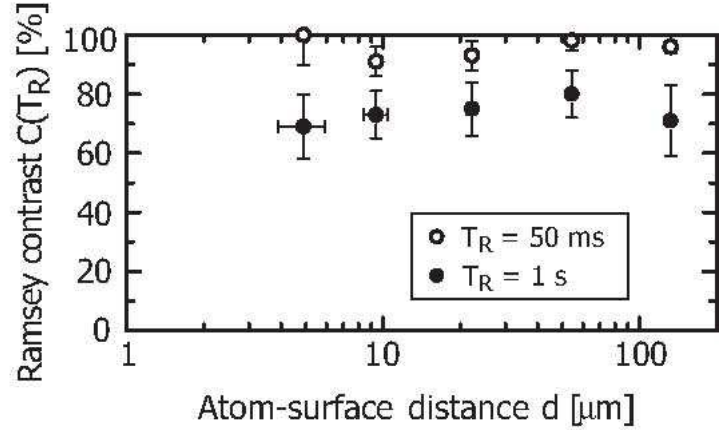

FIG. 7: Coherence lifetime measurements for the qubit state pair. (a) Ramsey spectroscopy of the $|0\rangle \leftrightarrow|1\rangle$ transition with atoms held at a distance $d=9 \mu \mathrm{m}$ from the chip surface. An exponentially damped sine fit to the Ramsey fringes yields a $1 / e$ coherence lifetime of $\tau_{c}=2.8 \pm 1.6 \mathrm{~s}$. Each data point corresponds to a single shot of the experiment. (b) Contrast $C\left(T_{R}\right)$ of the Ramsey fringes as a function of atom-surface distance $d$ for two values of the time delay $T_{R}$ between the $\pi / 2$-pulses. For each data point, $C\left(T_{R}\right)=\left(N_{\max }-N_{\min }\right) /\left(N_{\max }+N_{\min }\right)$ was obtained from a sinusoidal fit to frequency-domain Ramsey fringes. $N_{\max }\left(N_{\min }\right)$ is the maximum (minimum) of the oscillation in $N_{1}$.

compared the signal-to-noise ratio $S / N$ of the interference signal in the different traps. We typically observe $S / N=6$ for $T_{R}=1 \mathrm{~s}$, where $S$ is the peak-to-peak amplitude of the sinusoidal fit to the Ramsey oscillation and $N$ is the standard deviation of the fit residuals over one oscillation period. $S / N$ is independent of $d$ within experimental error, indicating that the processes causing amplitude and phase fluctuations of the interference signal do not depend on atom-surface distance on this time scale. The observed noise on the Ramsey oscillation is mostly phase noise and can be attributed to ambient magnetic field fluctuations, which are independent of atom-surface distance.

Our experiments show that the robust qubit state pair considered here can be manipulated on the atom chip with coherence lifetimes $\tau_{c}>1 \mathrm{~s}$ at distances down to a few microns from the chip surface. In the proposal for a quantum controlled phase gate on an atom chip [23], a gate operation time of $\tau_{g}=0.4 \mathrm{~ms}$ was estimated. Implementing this gate with our qubit state pair, $\tau_{c} / \tau_{g} \sim 10^{3}$ gate operations could be performed before decoherence from magnetic noise occurs. In contrast, in the original proposal of [23], the qubit is encoded in two states with a magnetic-field sensitive energy difference. The magnetic field sensitivity is more than a factor of $10^{3}$ higher than for our state pair, so that expected coherence lifetimes would be comparable to the gate operation time.

\section{State-dependent microwave potentials}

An implementation of the phase gate proposed in [23] with our qubit state pair requires potentials which affect the two qubit states differently. However, a combination of static magnetic and electric fields, as considered in [23, 29], does not provide state-selective potentials for our state pair, whose magnetic moments and electrostatic polarizabilities are equal. Optical potentials created by focussed laser beams with a frequency close to the D1 or D2 transition of ${ }^{87} \mathrm{Rb}$ are also impractical: if the detuning of the laser from the atomic resonance is much larger than the hyperfine splitting of the ${ }^{87} \mathrm{Rb}$ ground state, the resulting optical potentials are again nearly identical for the states $|0\rangle$ and $|1\rangle$. If, on the other hand, a detuning comparable to the hyperfine splitting is used, a differential optical potential could be created, but problems with decoherence due to spontaneous scattering of photons would arise.

To generate the state-dependent potential for our qubit, we propose to use microwave potentials in addition to static magnetic potentials on the atom chip [17]. Microwave potentials arise due to the AC Zeeman effect (the magnetic analog of the AC Stark effect) induced by tailored microwave near-fields. In ${ }^{87} \mathrm{Rb}$, microwave potentials derive from magnetic dipole transitions with a frequency near $\omega_{0} / 2 \pi=6.835 \mathrm{GHz}$ between the $F=1$ and $F=2$ hyperfine manifolds of the ground state. The magnetic component of a microwave field of frequency $\omega_{\mathrm{mw}}=\omega_{0}+\Delta$ couples the $\left|F=1, m_{1}\right\rangle$ to the $\left|F=2, m_{2}\right\rangle$ sublevels and leads to energy shifts that depend on $m_{1}$ and $m_{2}$. In a spatially varying microwave field, this results in a state-dependent potential landscape.

In Fig. 8 a, this situation is shown for a ${ }^{87} \mathrm{Rb}$ atom subject to a static magnetic field $\mathbf{B}_{0}(\mathbf{r})$, which defines the quantization axis, in combination with a microwave magnetic field $\mathbf{B}_{\mathrm{mw}}(\mathbf{r}) \cos \left(\omega_{\mathrm{mw}} t\right)$. The static field gives rise to the static Zeeman potential $U_{Z}(\mathbf{r})=\mu_{B} g_{F} m_{F}\left|\mathbf{B}_{0}(\mathbf{r})\right|$, which is identical for the qubit states $|0\rangle$ and $|1\rangle$, since for both states $g_{F} m_{F}=1 / 2$. For simplicity, we assume that $\mathbf{B}_{\mathrm{mw}}(\mathbf{r})$ is oriented parallel to the static field $\mathbf{B}_{0}(\mathbf{r})$, corresponding 
a)

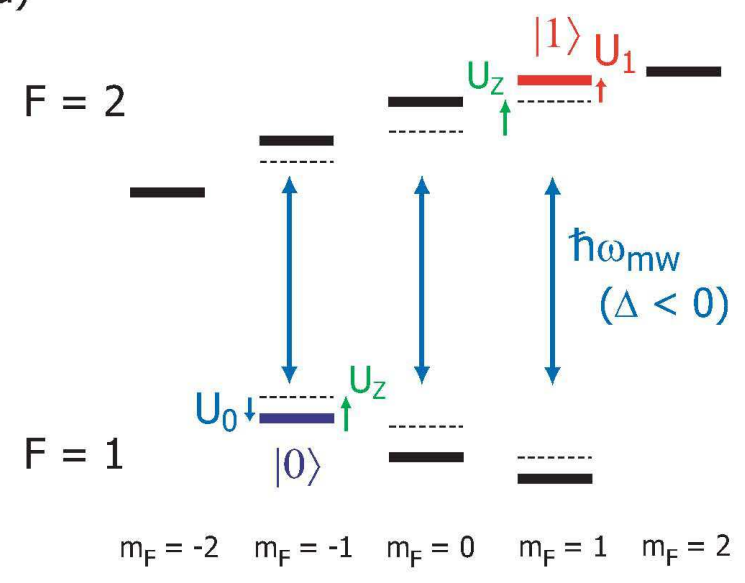

b)

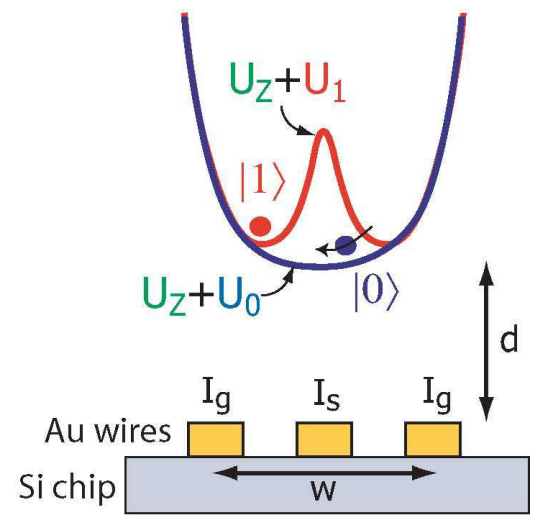

FIG. 8: State-dependent microwave potentials for the qubit states. (a) Energy-level diagram of the hyperfine structure of the ${ }^{87} \mathrm{Rb}$ ground state in a combined static magnetic and microwave field. $U_{Z}$ indicates the energy shift due to the static Zeeman effect, which is identical for $|0\rangle$ and $|1\rangle$. The magnetic field of the microwave couples the levels of $F=1$ to the levels of $F=2$, giving rise to energy shifts $U_{1}\left(U_{0}\right)$ for state $|1\rangle(|0\rangle)$, here shown for pure $\pi$ polarization and $\Delta<0$ (red detuning). This shift has opposite sign for $|0\rangle$ and $|1\rangle$. (b) Chip layout and state-dependent double well potential for a collisional phase gate on the atom chip. The three gold conductors form a coplanar waveguide of width $w$ for the microwave. $I_{s}\left(I_{g}\right)$ are the currents on the signal (ground) wires. The wires carry both stationary and microwave currents, see text. In combination, these currents create the potential $U_{Z}+U_{1}$ for state $|1\rangle$ and $U_{Z}+U_{0}$ for state $|0\rangle$ at a distance $d$ from the chip surface.

to pure $\pi$ polarization of the microwave. The microwave field thus couples the transitions $|0\rangle \leftrightarrow\left|F=2, m_{F}=-1\right\rangle$ and $\left|F=1, m_{F}=1\right\rangle \leftrightarrow|1\rangle$ with identical resonant Rabi frequencies $\Omega_{R}(\mathbf{r})=\sqrt{3 / 4} \mu_{B}\left|\mathbf{B}_{\mathrm{mw}}(\mathbf{r})\right| / \hbar$. In the limit of large detuning $\hbar|\Delta| \gg \hbar \Omega_{R}, U_{Z}$, the coupling leads to microwave potentials given by

$$
U_{1}(\mathbf{r})=-\frac{\hbar\left|\Omega_{R}(\mathbf{r})\right|^{2}}{4 \Delta} \text { and } \quad U_{0}(\mathbf{r})=\frac{\hbar\left|\Omega_{R}(\mathbf{r})\right|^{2}}{4 \Delta}
$$

for $|1\rangle$ and $|0\rangle$, respectively. Since the qubit state $|0\rangle$ belongs to $F=1$ while $|1\rangle$ belongs to $F=2$, the microwave potential has opposite sign for the two states, giving rise to the desired state-dependence of the potential.

In a combined static magnetic and microwave trap, in general both $\mathbf{B}_{0}(\mathbf{r})$ and $\mathbf{B}_{\mathrm{mw}}(\mathbf{r})$ vary with position. This leads to a position-dependent microwave coupling with in general all polarization components present. If $\hbar|\Delta| \gg$ $\hbar \Omega_{R}, U_{Z}$, the energy shifts due to the microwave coupling can be evaluated for each transition seperately. The overall magnetic microwave potential for the level $\left|F, m_{F}\right\rangle$ equals the sum of the energy shifts due to the individual transitions connecting to this level. The Zeeman splitting due to the static field (a few $\mathrm{MHz}$ ) prevents two-photon transitions between sublevels $m_{F}$ belonging to the same $F$ quantum number.

A trap for neutral atoms based on microwave potentials has been proposed in [30] and experimentally demonstrated in 31]. This trap employs microwave radiation in the far field of the source. Unlike in the case of optical radiation, which can be tightly focussed due to its short wavelength, the centimeter wavelength $\lambda_{\mathrm{mw}}$ of microwave radiation poses severe limitations on far-field traps: field gradients are very weak [31] and structuring the potential on the micrometer scale is impossible.

On atom chips, there is a natural solution to this problem [17]. The atoms are trapped at distances $d \ll \lambda_{\text {mw }}$ from the chip surface. Thus, they can be manipulated with microwave near fields, generated by microwave signals in on-chip transmission lines [32]. In the near field of the source currents and voltages, the microwave fields have the same position dependence as the static fields created by equivalent stationary sources. The maximum field gradients depend on the size of the transmission line conductors and on the distance $d$, not on $\lambda_{\mathrm{mw}}$. In this way, state-dependent microwave potentials varying on the micrometer scale can be realized. In combination with state-independent static magnetic microtraps, the complex potential geometries required for QIP can be realized.

The state-dependent double well potential needed for the phase gate proposed in 23] can be created with a chip layout as shown in Fig. 8 b. The three wires form a coplanar waveguide for the microwave. They carry both microwave and stationary currents, $I_{s}=I_{c}+I_{\mathrm{mw}} \cos \left(\omega_{\mathrm{mw}} t\right)$ and $I_{g}=I_{o}-\left(I_{\mathrm{mw}} / 2\right) \cos \left(\omega_{\mathrm{mw}} t\right)$. The stationary currents $I_{c}$ and $I_{o}$ flow in opposite directions and create a static magnetic double well potential at a distance $d$ from the chip surface, as 
discussed in [14]. We assume that the atoms are tightly confined in the transverse dimensions by a static magnetic potential created by additional wires not shown in the figure. The microwave currents create a microwave potential which is used to selectively remove the barrier of the double well for state $|0\rangle$, while increasing the barrier height for state $|1\rangle$ (Fig. $8 \mathrm{~b}$ ). Note that for $\Delta<0$, as in the figure, the labeling of the states $|1\rangle$ and $|0\rangle$ is interchanged compared to 23$]$.

To give a specific example, we consider atoms in a static-field trap at $d=1.8 \mu \mathrm{m}$ from a microwave guiding structure of size $w=d$ carrying a microwave signal of amplitude $I_{\mathrm{mw}}=15 \mathrm{~mA}$. A simulation of the microwave field yields a coupling with $\Omega_{R} / 2 \pi \sim 3.3 \mathrm{MHz}$ at the position of the static double well barrier, taking into account the magnetic microwave field of the signal wire and both ground wires. For $\Delta=10 \Omega_{R}$, the change in the static magnetic moment of the qubit states due to the coupling is of the order of $10^{-3}$, such that both states still experience approximately the same static magnetic potentials. The microwave, on the other hand, leads to a differential energy shift of $U_{1}-U_{0}=h \cdot 160 \mathrm{kHz}$, sufficiently large to remove the barrier for state $|0\rangle$. A detailed simulation for a realistic atom chip design shows that an improved version of the quantum phase gate of 23] can be implemented with our robust qubit state pair using microwave potentials on the atom chip. We find an overall gate fidelity of $F=0.996$ at a gate operation time of $\tau_{g}=1.1 \mathrm{~ms}[33]$, compatible with the requirements for fault-tolerant quantum computation.

\section{Qubit readout in microtraps}

The QIP schemes considered here use single atoms as qubit carriers, and thus the final readout requires single-atom detectivity. Again, the ability of atom chips to independently transport the individual qubit atoms is a key advantage: atoms can be brought close together for interaction, but spaced far apart and even transported to a remote detector for readout. This removes the optical resolution limitation that is still an unsolved problem in optical lattices. Thus, the basic requirement on an atom chip qubit detector is single-atom detectivity and compatibility with the presence of the chip. In our experiments, we have focused on optical detectors, where fast progress could be achieved. As an additional feature beyond single-atom detectivity, we have concentrated on detectors that will ultimately allow quantum non-demolition (QND) measurement of the number of atoms. A QND trapped-atom detector would only perturb the phase of the atomic state, but not, in particular, its vibrational energy in the trap. Therefore, such a detector could also be used in qubit preparation, for example in a "feedback loop" that prepares a single-atom state from a larger initial BEC, by combining it with a switchable loss mechanism.

To detect an atom optically, either absorption or dispersion can be used. The collection of fluorescence light from a single trapped atom is possible and has recently enabled remarkable experiments 34, 35]. However, the recoil from the spontaneously emitted fluorescence photons causes heating, ruling out the possibility of a QND measurement. It might seem that single-pass dispersive detection would offer a straightforward solution: the atom trap would be positioned in one arm of an interferometer, operating at a wavelength that is detuned far away from the atomic transitions. However, to reach the high sensitivity required for single-atom detection, a large number of photons must be sent through the interferometer, and it turns out that even this type of detection inevitably leads to spontaneous emission [36, 37]. The situation changes when an optical cavity is used to enhance the interaction of the atom with the optical field. In this case, single-atom detection with high signal-to-noise ratio is possible with less than one spontaneous emission on average, and improves with high cavity finesse $\mathcal{F}$ and small mode cross-section $w^{2}$.

This situation is adequately analyzed in the framework of cavity QED (cQED) 38]. The fundamental cavity QED parameters are the coherent atom-photon coupling rate $g_{0}$, the cavity damping rate $\kappa$ and the linewidth of the atomic transition $\gamma$. For single-atom detection, these parameters do not enter independently, but in the combination $C=g_{0}^{2} / 2 \kappa \gamma$ called the cooperativity parameter. The onset of the QND regime corresponds to $C>1$. Note that this condition is not identical with the strong coupling regime of $\mathrm{CQED}, g_{0}>\kappa, \gamma$. Indeed, QND detection is possible even in the regime of weak coupling.

To translate the cooperativity criterion $C>1$ into requirements on the cavity, it is instructive to analyze how $g_{0}$ and $\kappa$ relate to the design parameters of the cavity. For a symmetric Fabry-Perot (FP) cavity, these are the mirror radius of curvature $R$, the effective cavity length $d$, and the cavity finesse $\mathcal{F} \approx \pi /(T+\ell)$, where $T$ and $\ell$ are the transmission and losses of a single mirror. One finds

$$
\begin{aligned}
\kappa & \propto \mathcal{F}^{-1} d^{-1} \\
g_{0} & \propto d^{-3 / 4} R^{-1 / 4} \\
C & \propto(L R)^{-1 / 2}
\end{aligned}
$$


$C$ can be alternatively expressed as

$$
C=\frac{3 \lambda^{2} \mathcal{F}}{\pi^{3} w^{2}}
$$

where $w$ is the mode waist diameter (and we assume that the atoms are placed in this waist). This latter relation makes it intuitively clear why $C$ is the relevant parameter for single-atom detection efficiency: it is proportional to $\mathcal{F}$ (number of round-trips of a cavity photon), and inversely proportional to the mode waist diameter (which is to be compared to the atomic scattering cross-section $\sigma$ ). These relations hold within the stability range of the cavity, and as long as the mode diameter on the mirrors is small compared to the mirror diameter, so that clipping loss can be neglected.

For the extremely short, small-volume cavities that we consider here, $\gamma$ is always much smaller than $\kappa$ and $g_{0}$. Therefore, if the goal is to enter as far as possible into the strong-coupling regime, the cavity should optimize $g_{0} / \kappa$, i.e., increase the mirror distance $d$ towards the limit of the stability range. Indeed, for a given mirror curvature, $\kappa$ drops as $\kappa \propto d^{-1}$, whereas $g_{0}$ only decreases as $g_{0} \propto d^{-3 / 4}$, as long as $d \ll R$ : the ratio $g_{0} / \kappa$ increases with growing $d$ despite the decrease in the absolute value of $g_{0}$. By contrast, a cavity for single-atom detection should be designed to optimize the cooperativity $C$. According to the above proportionalities, this means that it should have a short length and small radius of curvature. A high finesse is obviously beneficial in both cases.

\section{Stable fiber Fabry-Perot cavities}

The "gold standard" for cQED cavities is still being set by macroscopic FP cavities with superpolished, concave mirrors. These mirrors have relatively large radii of curvature ( $R=20 \mathrm{~cm}$ is typical) and achieve record finesse values of $\mathcal{F}>2 \times 10^{6}$ [39]. However, these cavities are not compatible with a chip-based microtrap. The trap-surface distance is $\lesssim 250 \mu \mathrm{m}$, whereas the diameter of existing superpolished FP mirrors is at least $\sim 1 \mathrm{~mm}$, so that it would be extremely difficult to place the optical axis sufficiently close to the substrate surface and still maintain the tight mirror spacing required for high $C$. We have developed stable, fiber-based Fabry-Perot resonators (FFPs) [37] that avoid this problem. They employ concave dielectric mirror coatings with small radius of curvature, realized on the fiber tip. A stable cavity is constructed from two closely spaced fiber tips placed face-to-face (figure 9/a)). Thus, as an important difference to other microcavities such as microtoroid resonators (see for example [40]), the cavity mode is located in free space between the fibers, thus avoiding the extremely restrictive positioning requirements imposed by evanescent-field coupling.
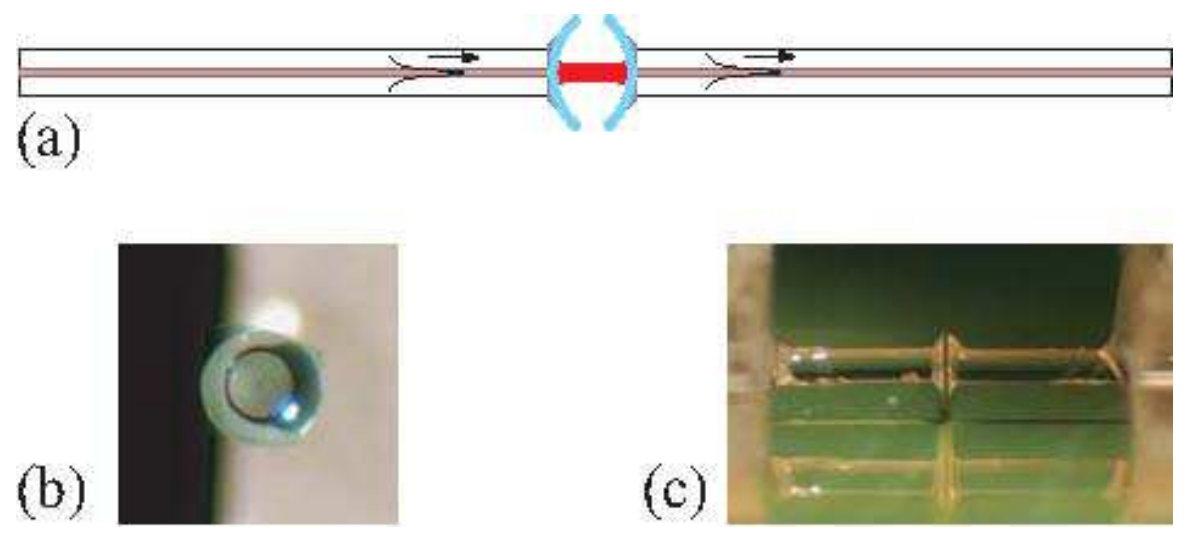

FIG. 9: (a) Concept of the stable FFP cavity. The basic building block is an optical fiber functionalized with a concave dielectric mirror. Two such fibers, brought sufficiently close to each other, result in a stable Fabry-Perot cavity which can be interrogated remotely, either in transmission or in reflection, through the two fibers (b) A single-mode optical fiber, total diameter $125 \mu \mathrm{m}$, processed with a concave mirror. The mirror has radius $1000 \mu \mathrm{m}$ with a stopband centered at $780 \mathrm{~nm}$. (c) A complete FFP cavity, realizing the configuration (a), mounted on an atom chip used in the detection of cold atoms (Fig. 11). 


\section{FFP cavity fabrication and performance}

We have fabricated stable, miniature FFP cavities using two different methods. Method 1 uses a commercially available lift-off coating [41]. The coating is produced on a convex template (we use a commercial ball lens), and then glued onto the fiber tip. After curing the transparent epoxy glue with UV light, the coating sticks to the fiber and a small force is enough to detach it from the ball lens template. The result is a fiber functionalized with a highly reflecting concave mirror, as shown in Fig. 9(b). A complete FFP cavity is shown in Fig. 9(c). This method reproducibly leads to cavity finesse values $\mathcal{F}>1000$ with modest experimental effort. We have used a cavity of this type to detect magnetically guided and trapped atoms, as described below. Method 2 employs laser surface processing to produce a low-roughness concave depression on the fiber tip, followed by multilayer coating using the ion beam sputtering technique. With this technique, we obtain finesse values $\mathcal{F} \sim 35000$ [4].

In both cases, because of the small fiber diameter $(125 \mu \mathrm{m})$, very short cavities $(<10 \lambda / 2)$ can be realized even when radii of curvature $R \leq 1 \mathrm{~mm}$ are used, still leaving a sufficiently large gap to introduce cold atoms. Let us consider the concrete example of a cavity that we have fabricated using method 1 . The mirror curvature is $R=1 \mathrm{~mm}$ and the distance $d=27 \mu \mathrm{m}$, leading to a mode volume $V_{m}=600 \mu \mathrm{m}^{3}$, to be compared to $V_{m}=1680 \mu \mathrm{m}^{3}$ for the smallest-volume macroscopic FP cavity that has been used with atoms [43]. In terms of cavity QED parameters, the small mode volume results in an exceptionally high coherent atom-photon coupling rate, $g_{0} / 2 \pi=180 \mathrm{MHz}$ (calculated for the Rb D2 line at $\lambda=780 \mathrm{~nm}$ ). Therefore, in spite of a comparatively high cavity damping rate $\kappa / 2 \pi=2.65 \mathrm{GHz}$, which results from the moderate finesse of the transfer coating and short cavity length, the cavity reaches a singleatom cooperativity parameter greater than unity, $C=g_{0}^{2} / 2 \kappa \gamma=2.1$, (for the Rb D2 line, $\gamma / 2 \pi=3.0 \mathrm{MHz}$ ) signaling the onset of quantum effects such as enhanced spontaneous emission into the cavity mode [38] and a significant modification of cavity transmission by the presence of a single atom.

Below we present an experiment in which we use the two-fiber cavity to detect an extremely small number of cold atoms magnetically trapped in the cavity using an atom chip. What is still missing is an improved absolute calibration of these results in order to determine whether they already realize, or only come close to single-atom detectivity. In any case, considering that the "method 2" cavities are now available and improve finesse by a factor 30, it seems clear that the problem of qubit detection can be solved using our FFP technology. Beyond QIP, we believe that this cavity type is also attractive for experiments exploiting the strong optical dipoles of semiconductor quantum dots, semiconductor nanocrystals and molecules, and for channel separation in telecommunication.

\section{On-chip atom detection with a FFP cavity}

We have detected magnetically trapped atoms with an FFP cavity on an atom chip [41]. The atoms are trapped on the chip and evaporatively cooled as in our previous experiments [17], but on a chip which incorporates the FFP resonator fabricated according to method 1 described above (Fig. 10). Trapped and guided atoms could be reproducibly detected in a great variety of experimental parameters and procedures. The cavity transmission signal allowed detection with good signal-to-noise ratio even when the atom number was far too small to be visible by our absorption-imaging camera system. A typical temperature of the atom cloud in the resonator was around $1 \mu \mathrm{K}$, with typical longitudinal and transverse trap frequencies around $100 \mathrm{~Hz}$ and $1 \mathrm{kHz}$, respectively. Clouds containing extremely few atoms were prepared using the RF knife, by applying repeated, rapid radiofrequency scans across the "trap bottom" frequency.

Figure 11] shows a cavity transmission spectrum recorded by scanning the probe laser across the D2 atomic transition for a fixed atom-cavity detuning of $\delta_{\text {cav }}=0$. Each point in the spectrum corresponds to a complete experimental sequence of preparation, evaporative cooling, positioning and detection. The atoms are initially trapped in the $|F=2, m=2\rangle$ ground state. The three transmission minima correspond to transitions from this state to the $F=1,2,3$ sublevels of the $5 p_{3 / 2}$ excited state. We have recorded such spectra for various $\delta_{\text {cav }}$ and for different mean atom numbers.

The parameter of interest is the minimum number $N_{\min }$ of atoms that must interact with the cavity mode in order to produce a detection signal with a good signal-to-noise ratio, say, 4 . The actual number of detected atoms is more difficult to extract from the measurements than with a macroscopic resonator. This is mainly due to large error bars on the measured on-resonance cavity transmission, which in turn are caused by the fact that incoupling mirror and fiber cannot be separated. For the resonator used in the experiment described here, the on-resonance transmission is in the permille range. This is due to an excessive number of layers in the dielectric mirror stack, applied by the coating manufacturer in an attempt to maximise the reflectivity. This problem no longer occurs with the cavities fabricated later according to method 2. In the experiments described here, however, the low transmission means that 


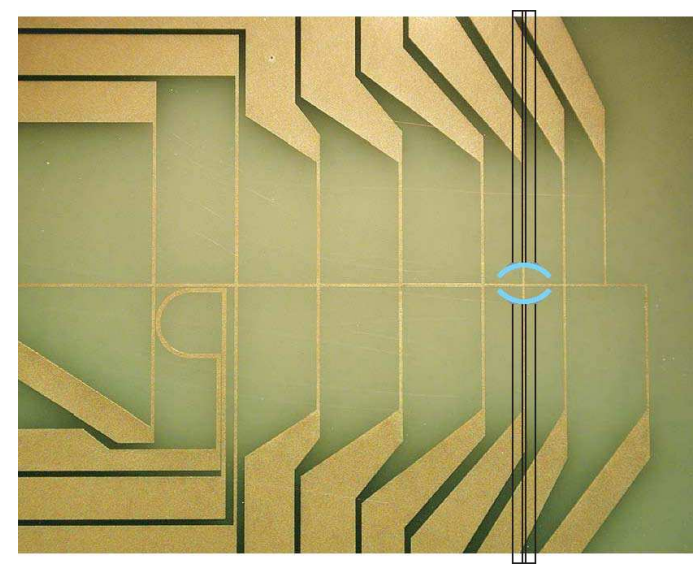

FIG. 10: The chip used for detecting magnetically trapped atoms by their interaction with a single optical mode in a fiber Fabry-Perot (FFP) cavity. The position of the cavity is indicated in exaggerated size for better visibility. Atoms are first trapped in the structures on the left side of the picture, then transported towards the cavity and evaporatively cooled in a multistep procedure and finally positioned in the resonator mode, where they are detected by the change in cavity transmission.

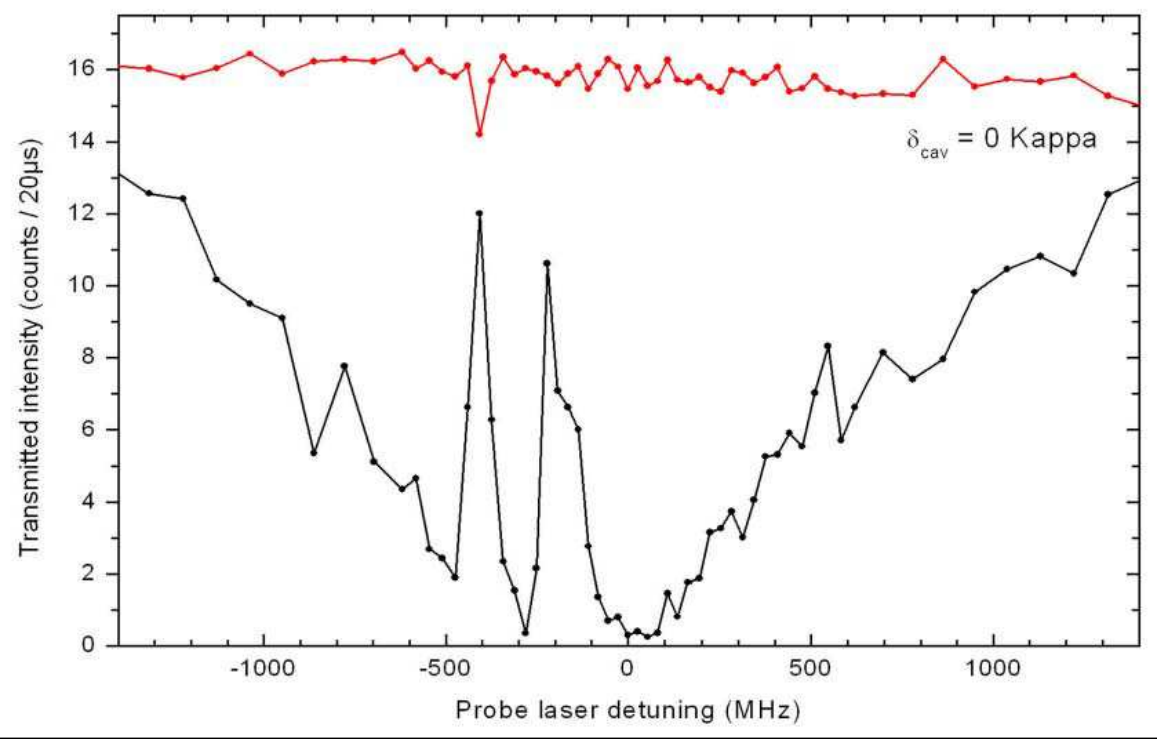

FIG. 11: Cavity tranmission spectrum without atoms (upper, red curve) and with atoms magnetically trapped in the onchip FFP cavity (lower, black curve). The atom-cavity detuning is fixed at $\delta_{\text {cav }}=0$. Each point corresponds to a complete experimental sequence of preparation, evaporative cooling, positioning and detection, for the atom-laser detuning indicated on the abscissa. Lines are to guide the eye. Zero probe laser detuning corresponds to the $F=2 \rightarrow F^{\prime}=3$ transition within the D2 multiplett.

for every detected photon, roughly $10^{3}$ photons have interacted with the atom without contributing to the signal. They do, however, contribute to heating due to spontaneous-emission, and we therefore expect the detectivity in this experiment to be limited by this spontaneous heating. Nevertheless, we expect $N_{\min }$ to be close to or below 1 .

From atom number measurement by absorption imaging, we can infer an upper limit of $N_{\min }$ which is of the order of 50 atoms. A much more precise value of $N_{\min }$ can be obtained from spectra such as in Fig. 11 These spectra were obtained with an FFP cavity of relatively low finesse $\mathcal{F} \sim 260$. This corresponds to a weak-coupling regime in which the atom-cavity interaction can be understood semiclassically. The spectra depend very strongly on the mean atom number in the cavity. We are now using a semiclassical model to fit the spectra, which will allow us to determine the actual number of intracavity atoms with good precision without the need to know the absolute cavity transmission. In this way, we will be able to determine the detectivity from the experimental results. 
These results demonstrate the suitability of FFP resonators for qubit readout on atom chips. The combination of this new cavity type with atom chips will enable new applications beyond atom detection. The laser-machined resonators, (method 2 described above), which we are now integrating into an atom chip experiment, reach a finesse $\mathcal{F} \sim 35000$, combined with an exceptionally small mode volume. For these resonators, with a mirror spacing of $d=25 \mu \mathrm{m}$, one obtains $g_{0} \sim 2 \pi \times 400 \mathrm{MHz}$ and $g_{0} / \kappa \sim 4$, entering the strong-coupling regime of cavity QED. But even in the regime of weak coupling, trapping an ultracold atom cloud in an optical cavity of high cooperativity, as demonstrated here, is a new experimental option which can radically simplify the implementation of high-fidelity atom-photon interfaces, for example in quantum communication [4].

\section{Single atom preparation}

With the advent of single-atom detectors on atom chips, it becomes possible to address the problem of deterministic single-atom preparation. For the QIP schemes considered here, each qubit is a single atom in the ground state of a magnetic potential. A first, simplistic approach is to start from a BEC and induce losses to reduce the atom number to an average value of 1 . With a QND detector, the actual number can be measured, and further reduced if necessary, with negligible excitation and loss. Nevertheless, this "trial-and-error" method becomes impractical for large numbers of qubits. Proposals for deterministic single atom preparation have been put forward in [45, 46]. The key element in these methods is a tightly confining potential, in which states with 1, 2 etc. atoms are energetically resolved due to the collisional interaction. A BEC serves as a reservoir from which single atoms can be repeatedly extracted in a deterministic way. Atom chips appear ideally suited to implement this idea, and we expect it to be experimentally realized within the next two years.

\section{CONCLUSION}

The fast experimental progress made with atoms in optical lattices and magnetic microtraps underlines the great potential of ultracold quantum gases for applications in QIP. In the experiments with optical lattices described here, a massively parallel quantum gate array was demonstrated for the first time [13], which allows the creation of a highly entangled many-body cluster state. In the future, it is important to explore quantum computing schemes which rely only on single-atom operations and measurements on the entangled many-body state. New theoretical developments show that even without the possibility of performing single-atom manipulations in the optical lattice, a quantum computer based on the controlled collisions demonstrated here could simulate a large class of complex Hamiltonians with translational invariance, which play an important role in condensed-matter physics.

A general quantum computer, however, requires the possibility to perform single-atom operations and measurements. The fiber Fabry-Perot resonators described here are an ideal system for achieving this goal. The detection of very small atom numbers was demonstrated in our experiments with a FFP resonator integrated on the atom chip 41. It seems clear that the problem of single qubit detection can be solved in the nearest future with the technical improvements of this detector which have been recently implemented [42]. We have furthermore shown that using a qubit state pair which is robust against magnetic-field fluctuations, coherence lifetimes exceeding one second can be achieved on an atom chip with atoms at distances down to a few microns from the chip surface [17]. Based on these developments, the main experimental challenges for the future are the reproducible preparation of single-atom states and the implementation of a quantum phase gate using microwave potentials on the atom chip. The theoretical fidelity of such a gate is 0.996 [33], compatible with the requirements for fault-tolerant quantum computation.

The success of these future experiments will determine whether QIP with neutral atoms is an advantageous alternative to other systems such as trapped ions, and allows the experimental investigation of even more complex problems such as quantum error correction.

This work has been funded by the Bavarian State Government (Kompetenznetzwerk Quanteninformation "A8"). Y.C. gratefully acknowledges support from the "CONQUEST" network (MRTN-CT-2003-505089).

\footnotetext{
† Present address: Laboratoire Kastler Brossel de l'E.N.S, 24 Rue Lhomond, 75231 Paris Cedex 05, France

¥ Present address: Dept. of Physics UCB/JILA, Boulder, CO 80309-0440, U.S.A.

$\S$ Present address: Varian Physics Building, Stanford University, Stanford, CA 94305, U.S.A.

ฯ Present address: Harvard University, Department of Physics, Cambridge, MA 02138, U.S.A.
} 
** Present address: Institut für Physik, Johannes Gutenberg-Universität, 55099 Mainz, Germany

[1] D. Jaksch et al., Phys. Rev. Lett. 81, 3108 (1998).

[2] M. Greiner et al., Nature 415, 39 (2002).

[3] G. K. Brennen et al., Phys. Rev. Lett. 82, 1060 (1999).

[4] H.-J. Briegel et al., J. Mod. Opt. 47, 415 (2000).

[5] R. Raussendorf and H.-J. Briegel, Phys. Rev. Lett. 86, 5188 (2001).

[6] G. K. Brennen, I. H. Deutsch, and C. J. Williams, Phys. Rev. A 65, 022313 (2002).

[7] D. Jaksch et al., Phys. Rev. Lett. 82, 1975 (1999).

[8] H.-J. Briegel and R. Raussendorf, Phys. Rev. Lett. 86, 910 (2001).

[9] A. Sørensen and K. Mølmer, Phys. Rev. Lett. 83, 2274 (1999).

[10] W. Dür et al., Phys. Rev. A 66, 052319 (2002).

[11] E. Jané et al., Quant. Inf. Comp. 3, 15 (2003).

[12] O. Mandel et al., Phys. Rev. Lett. 91, 010407 (2003).

[13] O. Mandel et al., Nature 425, 937 (2003).

[14] J. Reichel, Appl. Phys. B 74, 469 (2002).

[15] R. Folman, P. Krüger, J. Schmiedmayer, J. Denschlag, and C. Henkel, Adv. At. Mol. Opt. Phys. 48, 263 (2002).

[16] D. P. DiVincenzo, Fortschr. Phys. 48, 771 (2000).

[17] P. Treutlein, P. Hommelhoff, T. Steinmetz, T. W. Hänsch, and J. Reichel, Phys. Rev. Lett. 92, 203005 (2004).

[18] P. Hommelhoff, W. Hänsel, T. Steinmetz, T. W. Hänsch, and J. Reichel, New J. Phys. 7, 3 (2005).

[19] T. Schumm, S. Hofferberth, L. M. Andersson, S. Wildermuth, S. Groth, I. Bar-Joseph, J. Schmiedmayer, and P. Krüger, Nature Physics 1, 57 (2005).

[20] B. Lev, Quant. Inf. Comp. 3, 450 (2003).

[21] S. Groth, P. Krüger, S. Wildermuth, R. Folman, T. Fernholz, J. Schmiedmayer, D. Mahalu, and I. Bar-Joseph, Appl. Phys. Lett. 85, 2980 (2004).

[22] A. S. Sørensen, C. H. van der Wal, L. I. Childress, and M. D. Lukin, Phys. Rev. Lett. 92, 063601 (2004).

[23] T. Calarco, E. A. Hinds, D. Jaksch, J. Schmiedmayer, J. I. Cirac, and P. Zoller, Phys. Rev. A 61, 022304 (2000).

[24] F. Schmidt-Kaler, S. Gulde, M. Riebe, T. Deuschle, A. Kreuter, G. Lancaster, C. Becher, J. Eschner, H. Häffner, and R. Blatt, J. Phys. B: At. Mol. Phys. 36, 623 (2003).

[25] C. Henkel, P. Krüger, R. Folman, and J. Schmiedmayer, Appl. Phys. B 76, 173 (2003).

[26] D. M. Harber, H. J. Lewandowski, J. M. McGuirk, and E. A. Cornell, Phys. Rev. A 66, 053616 (2002).

[27] W. Hänsel, P. Hommelhoff, T. W. Hänsch, and J. Reichel, Nature 413, 498 (2001).

[28] T. R. Gentile, B. J. Hughey, D. Kleppner, and T. W. Ducas, Phys. Rev. A 40, 5103 (1989).

[29] P. Krüger, X. Luo, M. W. Klein, A. Brugger, A. Haase, S. Wildermuth, S. Groth, I. Bar-Joseph, R. Folman, and J. Schmiedmayer, Phys. Rev. Lett. 91, 233201 (2003).

[30] C.C. Agosta et al., Phys. Rev. Lett. 62, 2361 (1989).

[31] R. J. C. Spreeuw, C. Gerz, L. S. Goldner, W. D. Phillips, S. L. Rolston, and C. I. Westbrook, Phys. Rev. Lett. 72, 3162 (1994).

[32] R. E. Collin, Foundations for Microwave Engineering, 2nd ed. (John Wiley and Sons, Hoboken, 2001).

[33] P. Treutlein, T.W. Hänsch, J. Reichel, A. Negretti, M.A. Cirone, and T. Calarco, to be published (2006).

[34] J. Volz, M. Weber, D. Schlenk, W. Rosenfeld, J. Vrana, K. Saucke, H. Kurtsiefer, and H. Weinfurter, Phys. Rev. Lett. 96, 030404 (2006).

[35] J. Beugnon, M. P. A. Jones, J. Dingjan, B. Darquié, G. Messin, A. Browaeys, and P. Grangier, Nature 440, 779 (2006).

[36] J. E. Lye, J. J. Hope, and J. D. Close, Phys. Rev. A 67, 043609 (2003).

[37] R. Long, T. Steinmetz, P. Hommelhoff, W. Hänsel, T. W. Hänsch, and J. Reichel, Phil. Trans. R. Soc. Lond. A 361, 1375 (2003).

[38] H. J. Kimble, Phys. Scr. T76, 127 (1998).

[39] G. Rempe, R. J. Thompson, H. J. Kimble, and R. Lalezari, Opt. Lett. 17, 363 (1992).

[40] S. M. Spillane, T. J. Kippenberg, K. J. Vahala, K. W. Goh, E. Wilcut, and H. J. Kimble, Phys. Rev. A 71, 013817 (2005).

[41] T. Steinmetz, Y. Colombe, G. Dubois, T. W. Hänsch, and J. Reichel, to be published (2006).

[42] D. Hunger, T. Steinmetz, T. W. Hänsch, and J. Reichel, to be published (2006).

[43] C. J. Hood, T. W. Lynn, A. C. Doherty, A. S. Parkins, and H. J. Kimble, Science 287, 1447 (2000).

[44] L.-M. Duan, M. D. Lukin, J. I. Cirac, and P. Zoller, Nature 414, 413 (2001).

[45] R. B. Diener, B. Wu, M. G. Raizen, and Q. Niu, Phys. Rev. Lett. 89, 070401 (2002).

[46] B. Mohring, M. Bienert, F. Haug, G. Morigi, W. P. Schleich, and M. G. Raizen, Phys. Rev. A 71, 053601 (2005). 